
\title{
Tissue-agnostic drug approvals: how does this apply to patients with breast cancer?
}

\author{
Luiza N. Weis ${ }^{1}$, Sara M. Tolaney $\mathbb{D}^{2,3}$, Carlos H. Barrios $\mathbb{1}^{4}$ and Romualdo Barroso-Sousa $\mathbb{i D}^{1,5 凶}$
}

Precision medicine has provided new perspectives in oncology, yielding research on the use of targeted therapies across different tumor types, regardless of their site of origin, a concept known as tissue-agnostic indication. Since 2017, the Food and Drug Administration (FDA) has approved the use of three different agents for tumor-agnostic treatment: pembrolizumab (for patients with microsatellite instability or high tumor mutational burden) and larotrectinib and entrectinib (both for use in patients harboring tumors with NTRK fusions). Importantly, the genomic alterations targeted by these agents are uncommon or rare in breast cancer, and little information exists regarding their efficacy in advanced breast cancer. In this review, we discuss the prevalence of these targets in breast cancer, their detection methods, the clinical characteristics of patients whose tumors have these alterations, and available data regarding the efficacy of these agents in breast cancer.

npj Breast Cancer (2021)7:120; https://doi.org/10.1038/s41523-021-00328-3

\section{INTRODUCTION}

Breast cancer $(B C)$ represents the most frequently occurring cancer and is the leading cause of cancer-related deaths among female patients worldwide ${ }^{1}$. Although substantial progress in treatment has been made, metastatic breast cancer (MBC) remains an incurable disease, and the 5-year survival rate for stage IV breast cancer is approximately $25 \%{ }^{2}$.

With the emergence of next-generation sequencing (NGS) tools, cancer genomic data have become more widely available, and cancer therapy has shifted from a purely histology-based approach toward incorporating a precision medicine-based approach. Novel oncogenic drivers and biomarkers have been described across different tumor types, leading to the development of targeted therapies and the design of innovative trials, many of which are evaluating tissueagnostic therapies ${ }^{3}$.

The first tissue-agnostic treatment approval was granted by the FDA to pembrolizumab in patients with high microsatellite instability (MSI-H) tumors in 2017, followed by larotrectinib and entrectinib for the treatment of cancers harboring NTRK fusions in 2018 and 2019, respectively ${ }^{4}$. In 2020, the FDA expanded pembrolizumab approval to a new tissue-agnostic indication: high tumor mutational burden (TMB-H) ${ }^{5}$ (Fig. 1).

While these approvals were based on data from phase I and II trials conducted across several tumor histologies, only a few patients with MBC were included in those studies ${ }^{6-8}$. Therefore, we present an overview of the prevalence, detection methods, clinical characteristics, and data regarding the treatment efficacy of drugs targeting MSI-H/dMMR, NTRK fusions, and TMB-H in breast cancer. Finally, we also discuss the incorporation of these three biomarker tests and associated targeted therapies for $M B C$ into routine clinical practice, including recommendations of medical societies and evidence of utility according to the ESMO Scale for Clinical Actionability of Molecular Targets (ESCAT) scale 9 .

\section{MISMATCH REPAIR DEFICIENCY (DMMR) AND HIGH MICROSATELLITE INSTABILITY (MSI-H)}

Microsatellites are repeated sequences of 1-6 nucleotides and are mostly located near the ends of chromosomes. They are particularly susceptible to acquired errors when the mismatch repair (MMR) system is impaired ${ }^{10}$. The MMR system represents one of the DNA repair pathways and corrects DNA base substitution mismatch, insertion or deletion, and slippageconditions generated by DNA replication errors ${ }^{11}$. MMR deficiency (dMMR) arises due to mutations in at least one of the genes that encodes proteins in the MMR system (MLH1, MSH2, MSH6, and PMS2) or through methylation of the $M L H 1$ gene promoter that leads to $\mathrm{MSI}$ through accumulations of errors in DNA microsatellites $^{12}$.

\section{How to determine MSI/MMR status}

Tumor MSI/MMR status can be tested using immunohistochemistry $(\mathrm{IHC})$, polymerase chain reaction (PCR) and, more recently, NGS techniques (Fig. 2).

IHC for the MMR proteins MLH1, MSH2, MSH6, and PMS2 is a practical and widely available methodology among pathology laboratories. AMMR by IHC is defined by the absence of nuclear staining of some of the abovementioned MMR proteins in the tumor with preserved internal positive cell controls ${ }^{10}$. If $\mathrm{IHC}$ expression of at least one of those proteins is lost, the diagnosis of dMMR is established. Importantly, while there is a strong recommendation for performing $\mathrm{IHC}$ for all tumors belonging to the spectrum of Lynch syndrome, there are insufficient data for definitive recommendations in breast cancer. In cases of indeterminate IHC results, a molecular test preferentially based on PCR is indicated ${ }^{13}$.

The assessment of microsatellite alterations using classical PCRbased methodology combined with fragment length analysis is usually performed with DNA samples from tumors and paired normal DNA. This can be done using two panels: The National Cancer Institute $(\mathrm{NCl})$ panel, which evaluates two single nucleotide repeat loci, BAT-25 and BAT-26, and three dinucleotide repeat loci,

${ }^{1}$ Instituto de Ensino e Pesquisa Hospital Sírio-Libanês, São Paulo-SP, Brazil. ${ }^{2}$ Department of Medical Oncology, Dana-Farber Cancer Institute, Boston, MA, USA. ${ }^{3}$ Harvard Medical School, Boston, MA, USA. ${ }^{4}$ LACOG, Oncoclinicas, Porto Alegre, Brazil. ${ }^{5}$ Oncology Center, Hospital Sírio-Libanês Brasília, Brasília-DF, Brazil. ${ }^{\circledR}$ email: romualdo.barroso@hsl.org.br 


\section{NTRK fusions}

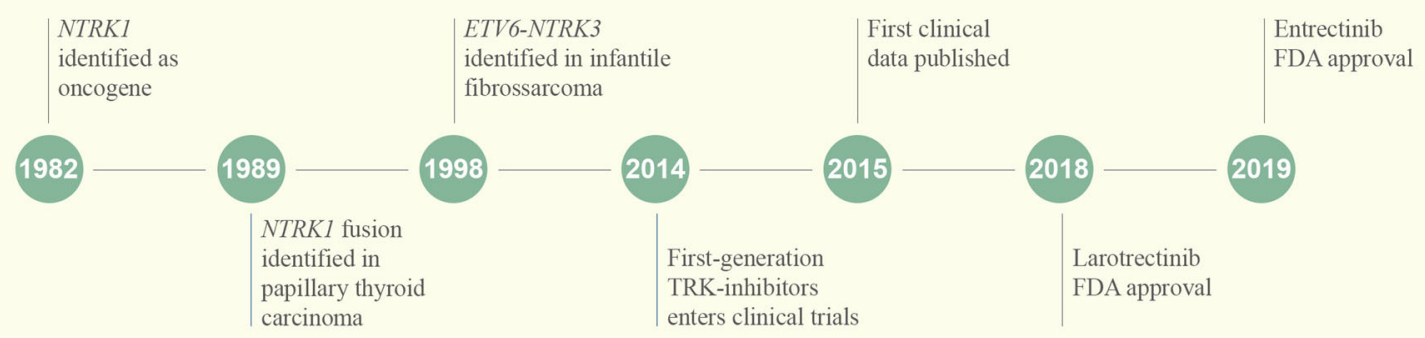

\section{MSI-H/dMMR}

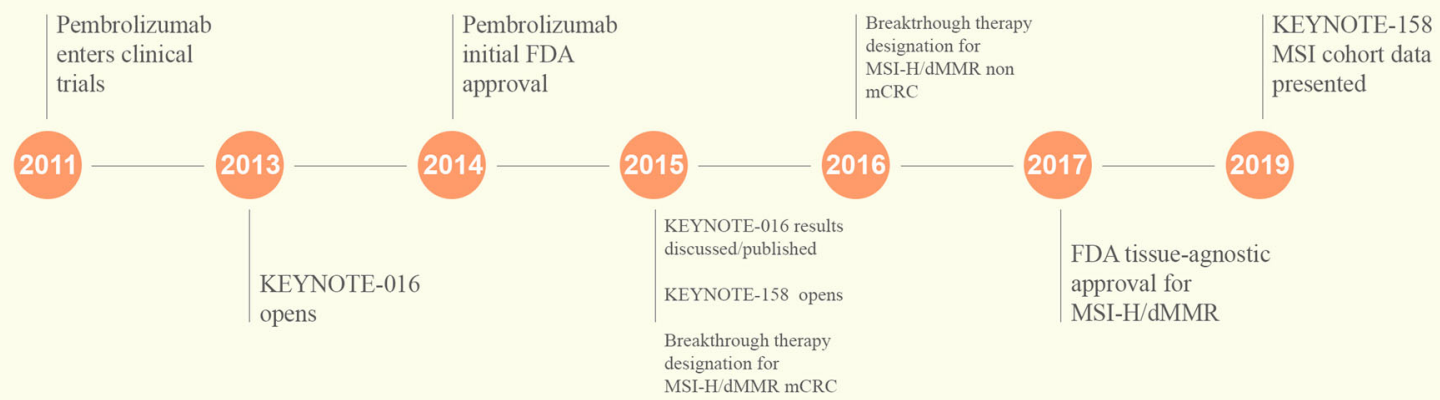

TMB-H

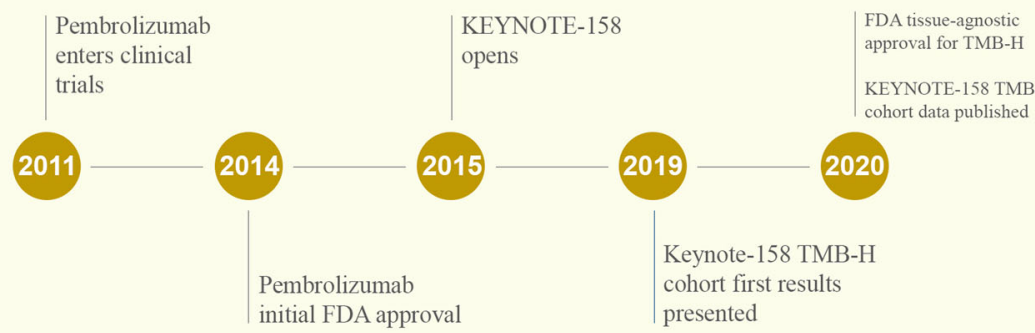

Fig. 1 Timeline of discovery, validation, and clinical development of tissue-agnostic therapies. dMMR: mismatch repair deficiency, FDA: The United States Food and Drug Administration agency; MSI-H: microssatellite instability high, mCCR: metastatic colorectal cancer, TMB-H: tumor mutational burden high (cut-off $\geq 10$ mutations/megabase).

D2S123, D5S346 and D17S250; and pentaplex PCR, using five polyA mononucleotide repeats (BAT-25, BAT-26, NR-21, NR-24, and NR27). Tumors with instability at 2 or more of these markers are defined as MSI- $\mathrm{H}^{10,12,14,15}$. In fact, pentaplex PCR is the preferred panel given its higher sensitivity and specificity ${ }^{13,16}$. Frequently, clinical trials that assessed MSI/MMR status used both IHC and PCR methodologies.

More recently, the use of NGS panels has been validated for MSI-H diagnosis, and the reported concordance rates between NGS testing and IHC or PCR are both high ${ }^{17,18}$. While this can potentially become an alternative molecular test, with the advantage of simultaneous testing of TMB and other actionable gene mutations and optimization of tumor tissue usage, it is important to highlight that these tests should be performed at validated laboratories.

\section{Approval of pembrolizumab for treating MSI-H/dMMR cancers} Given that most MSI-H or dMMR tumors exhibit a hypermutated phenotype ( $>10$ mutations per megabase) and that tumors with TMB-H present higher numbers of neoantigens that can potentially activate antitumor immunity, a hypothesis emerged suggesting that MSI-H tumors would be more suitable for immunotherapy.

In 2015, Le et al. published the results of KEYNOTE-016, a phase II trial designed to evaluate the clinical activity of pembrolizumab in 41 patients with treatment-refractory progressive metastatic carcinoma with or without dMMR. Pembrolizumab treatment resulted in increased objective response rates (ORRs) and superior progression-free survival (PFS) for dMMR colorectal and noncolorectal cancer patients compared to MMR-proficient colorectal cancer patients ${ }^{19}$. In an expanded phase of the abovementioned study, 86 patients with advanced dMMR metastatic solid tumors (across 12 different tumor types) who had been treated with at least one prior therapy received pembrolizumab. The primary endpoint, ORR, was 53\%, and a complete response was achieved by $21 \%$ of patients ${ }^{20}$.

In May 2017, based on data from five studies, including the KEYNOTE-016, the FDA granted accelerated approval to pembrolizumab for the treatment of patients with advanced or metastatic MSI-H or dMMR colorectal cancer progressing on conventional 


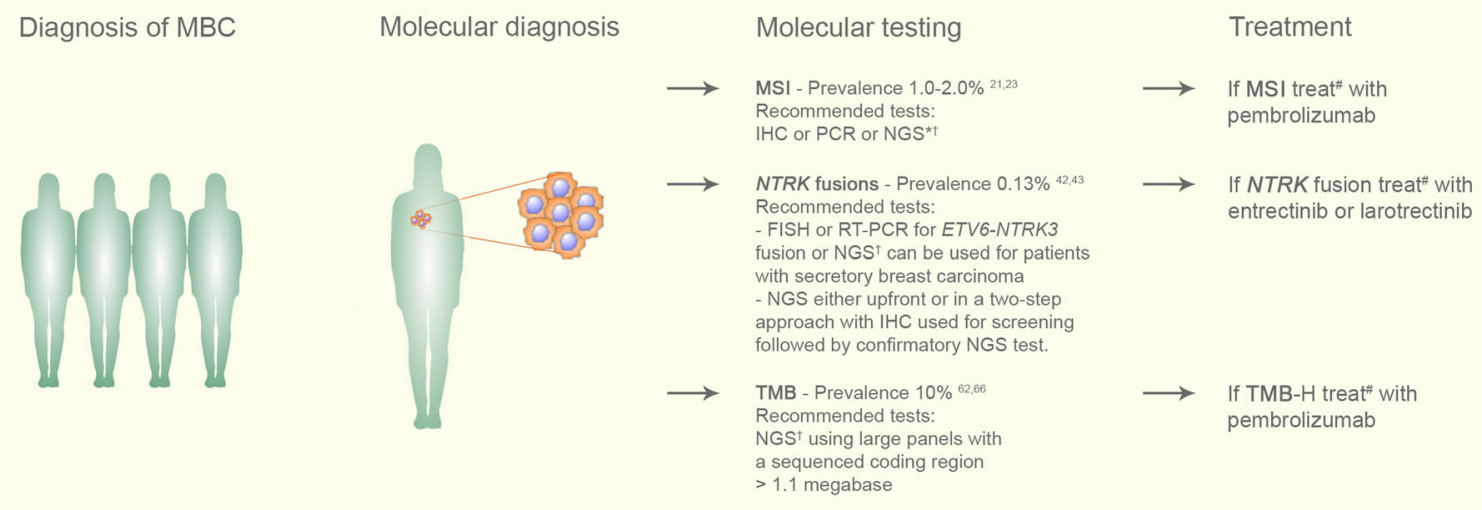

Fig. 2 Suggested flowchart for testing and using approved tumor-agnostic therapies in patients with metastatic breast cancer. Following the diagnosis of metastatic breast cancer, formalin-fixed, paraffin-embedded (FFPE) tissue blocks from archival tissue or from a new tumor biopsy should be used to evaluate the status of one of the three discussed biomarkers: MSI, TMB-H, or NTRK fusions. In the case of TMB, blood samples can be collected to perform liquid biopsy-based NGS panels. For those patients harboring any of these three biomarkers who have progressed following prior treatment and who have no satisfactory alternative treatment options, we recommend the use of the appropriate tissue-agnostic approved therapy. IHC: immunohistochemistry; FFPE: formalin-fixed, paraffin-embedded; FISH: fluorescence in situ hybridization; MBC: metastatic breast cancer; NGS: next-generation sequencing; PCR: polymerase chain reaction. ${ }^{*}$ In cases of negative IHC for dMMR in breast cancer, confirmatory PCR or NGS could be performed. ${ }^{\dagger} \mathrm{NGS}$ should be performed preferentially at validated laboratories. \#Treatment with the designated agnostic therapy should be started during the course of therapy for patients with the target biomarker who have progressed following prior treatment, and no satisfactory alternative treatment options are available.

therapy and other solid tumors also progressing after prior therapy without satisfactory alternative treatment options ${ }^{5.12}$.

More recently, data from the dMMR cohort of the KEYNOTE-158, a nonrandomized, open-label, multicohort, phase 2 study designed to evaluate pembrolizumab and predictive biomarkers in patients with advanced solid tumors, were presented. Overall, 233 patients with previously treated, advanced dMMR/MSI-H noncolorectal cancers who received pembrolizumab were included. After a median follow-up of 13.4 months, the ORR as the primary endpoint was $34.3 \%$, the median PFS was 4.1 months, and the median OS was 23.5 months ${ }^{6}$.

\section{MSI-H/dMMR in breast cancer}

Furthermore, little is known about the clinical features or prognostic value of MSI-H/dMMR in breast cancer as well as its association with response to ICls (immune checkpoint inhibitors). Overall, the prevalence of MSI-H/dMMR in breast cancer is less than $2 \%{ }^{21-23}$.

Fusco et al. evaluated the clinical value of MMR IHC and MSI analysis in 444 surgical breast cancer specimens, reporting $15 \%$ of dMMR tumors by IHC. When these tumors were analyzed by PCR with the five markers recommended by the National Cancer Institute, 91\% were microsatellite-stable, suggesting that these methods might not be interchangeable in breast cancer ${ }^{24}$. Addressing the usefulness of MSI analysis in breast cancer, Siah and colleagues performed a tabulated survey that included 18,055 microsatellite analyses and found that greater than 300 different microsatellite markers were used to detect MSI in breast cancer. By restricting the survey to larger studies (>100 DNA samples analyzed), D11S988 was the most informative microsatellite marker, with a MSI-positive detection rate of $17.7 \%{ }^{25}$. Such results stress the importance of performing studies to evaluate the concordance between different methods for identifying a tissueagnostic biomarker.

A retrospective study in 228 Japanese patients with primary triple-negative breast cancer (TNBC) revealed a prevalence of $0.9 \%$ (two cases) ${ }^{26}$. Another retrospective study found four (1.8\%) MSI$\mathrm{H} / \mathrm{dMMR}$ cases by IHC among 226 TNBC patients and none in 90 non-TNBC patients ${ }^{27}$. One tumor was classified as MSI-H/dMMR by PCR analysis, exhibiting hypermethylation of the MLH1 promoter.
Notably, all four patients diagnosed with BC were over 50 years of age, had a family history of colorectal cancer, presented with highgrade tumors, and had invasive ductal carcinoma ${ }^{27}$. Horimoto and colleagues also evaluated MSI-H/dMMR in TNBC but focused on its relationship with tumor-infiltrating lymphocytes (TILs), especially in medullary carcinomas-a histological type enriched with TILs. Among 101 samples (63 classified as TIL-high TNBC and 38 as medullary carcinoma), there were no MSI-H/dMMR tumors ${ }^{28}$. Interestingly, unlike mucinous carcinoma of the colon, ovary, and endometrium, Lacroix-Triki et al. did not show an association between mucinous carcinoma of the breast and $\mathrm{MSI}^{29}$. Taken together, these data indicate that there are no clinical or pathological features in breast cancer that can be correlated with MSI.

Importantly, there is a paucity of data regarding the efficacy of pembrolizumab in patients with breast cancer harboring MSI-H/ dMMR. Among 149 patients recruited from those five cohorts that provided data for pembrolizumab approval in MSI-H/dMMR metastatic cancers, only two had breast cancer, and both achieved partial responses, with durations of 7.6 and 15.9 months, respectively (Table 1$)^{5}$. In KEYNOTE-158, five breast cancer patients were included in this trial, but their clinical characteristics and outcomes are unknown (Table 1$)^{5}$. No patients with breast cancer were included in KEYNOTE-016 ${ }^{19,20}$.

\section{NEUROTROPHIC TROPOMYOSIN RECEPTOR KINASE (NTRK) FUSIONS}

The NTRK1, NTRK2, and NTRK3 genes encode the neurotrophin receptors TRKA, TRKB, and TRBC, respectively, which are predominantly transcribed in the nervous system in adult tissues. The TRK family plays an important role in nervous system development through regulation of cell proliferation, differentiation, apoptosis, and survival of neurons in both central and peripheral nervous systems ${ }^{30}$.

Fusions involving these genes are the most common mechanisms of oncogenic TRK activation and are found in both adult and pediatric tumors. NTRK fusions are enriched in rare cancer types, including infantile fibrosarcoma, congenital mesoblastic nephroma, secretory breast carcinoma, and mammary analog secretory carcinoma. Common tumors, such as lung, melanoma, 


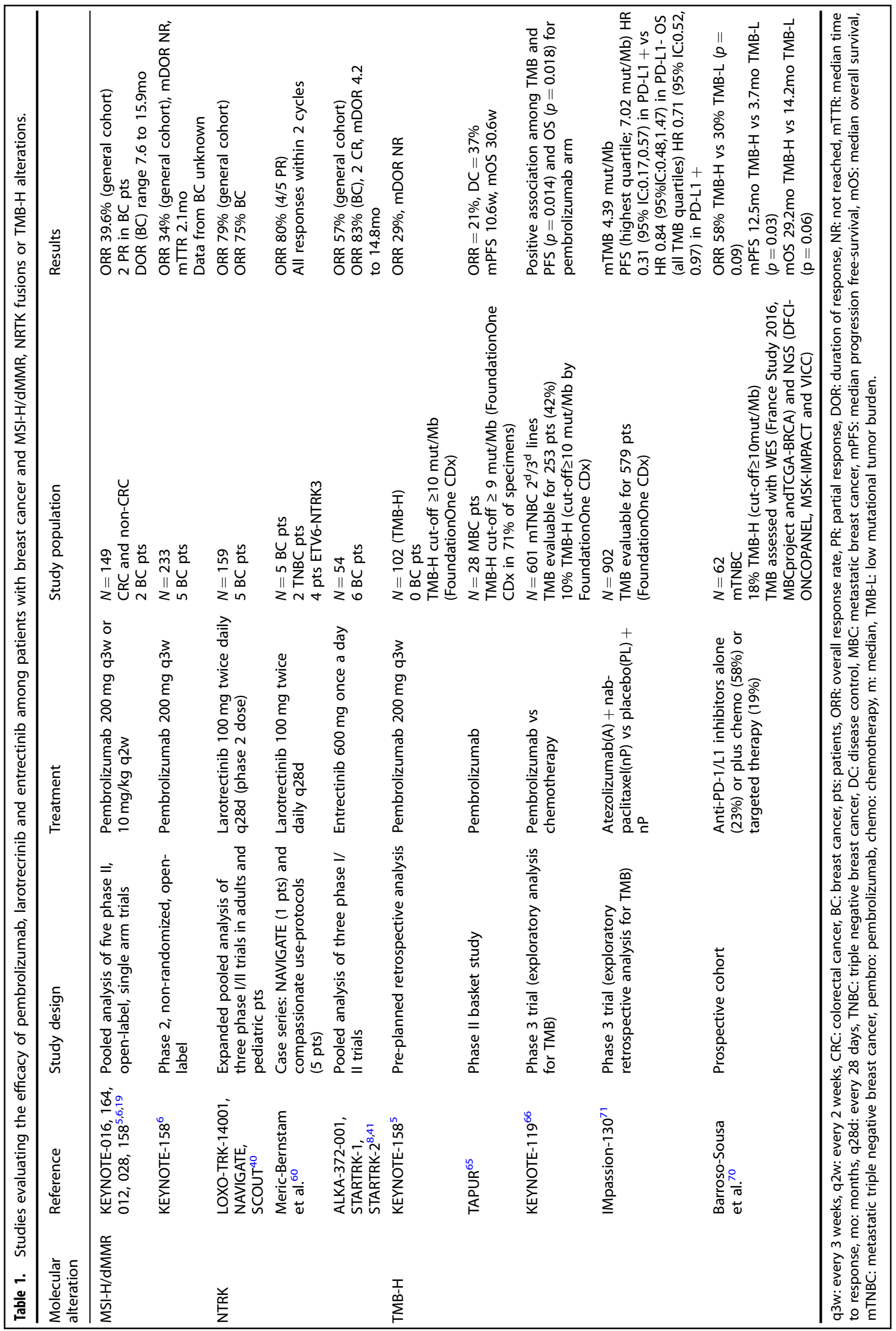


and colorectal cancers, have low frequencies of these genomic alterations ${ }^{31}$.

\section{How to determine NTRK fusions status}

There are different methods for identifying NTRK fusions: fluorescence in situ hybridization (FISH), RT-PCR, and RNA- or DNA-based $\mathrm{NGS}^{32}$. Notably, IHC may be used as a screening method, as we will discuss below (Fig. 2).

The use of FISH or RT-PCR is not recommended as a screening tool and should be reserved for cases where NTRK fusions are highly recurrent (ETV6-NTRK3 fusion) as in the case of infantile fibrosarcoma or secretory breast carcinoma ${ }^{33}$. FISH can be performed with break-apart probes for the three NTRK genes, which requires either separate or multiplex assays, or through a break-apart probe for the ETV6 gene in cases that are histologically suggestive of ETV6-NTRK3 fusions. FISH is not able to identify the gene fusion partner, requires expertise, and is more expensive when a multiplex assay is used. RT-PCR provides direct evidence of a NTRK fusion and detects only known fusion partners and breakpoints $^{15,32}$.

RNA- or DNA-based NGS methods are able to assess NTRK fusions with the advantage of providing other important molecular information, including the presence of other oncogenic drivers, tumor mutation burden, and monitoring of patients for the development of resistance mutations. RNA-based NGS has some advantages over DNA, since it is an approach that allows de novo detection of gene fusion transcripts that have not been previously described and increases the sensitivity of detection in low tumor purity samples ${ }^{33}$.

For tumors that rarely harbor NTRK fusions, front-line NGS-based approaches or a two-step approach with IHC followed by sequencing tests are indicated ${ }^{32}$. IHC examines the expression of TRK proteins but does not directly detect NTRK fusions ${ }^{34,35}$. It can be performed through different antibodies, such as antibodies directed against specific NTRK proteins or a pan-TRK antibody cocktail $^{32}$. Tumors are considered positive if $\geq 1 \%$ of tumor cells exhibit positivity at any intensity above the background ${ }^{36}$. The staining pattern is variable in intensity and localization (nucleus, cytoplasm, or membrane), showing a correlation with the fusion partner ${ }^{34}$. IHC has shown high sensitivity (from $93 \%^{34}$ to $100 \%^{37}$ ) and specificity (from $93 \%{ }^{37}$ to $100 \%^{34}$ ) for the detection of NTRK fusions. Consequently, IHC, when positive, may be used to enrich patients with NTRK fusions as part of a two-step detection process. Importantly, IHC shows lower sensitivity for NTRK3 fusions and lower specificity for tumors with neuronal and muscular differentiation ${ }^{38}$.

\section{Approval of larotrectinib and entrectinib for treating tumors with NTRK fusion}

In November 2018, the FDA granted accelerated approval for larotrectinib-a potent and selective inhibitor of all three TRK proteins-for adult and pediatric patients with solid tumors harboring NTRK gene fusion without a known acquired resistance mutation with metastatic disease or for whom surgical resection would likely result in severe morbidity, and who had no satisfactory alternative treatments available or whose cancer had progressed following treatment ${ }^{39}$. This approval was based on the results of three multicenter, open-label, single-arm clinical trials that evaluated 55 patients treated with larotrectinib and demonstrated an ORR of $75 \%$, with median duration of response and PFS not reached ${ }^{7}$. In an expanded pooled efficacy analysis of 159 patients recently published, the ORR was $79 \%$, and complete responses were achieved in $16 \%$ of patients with a median PFS of 28.3 months and median OS of 44.4 months ${ }^{40}$.

In 2019, another NTRK inhibitor, entrectinib, was granted FDA approval for the same therapeutic indications as larotrectinib. Data from a pooled analysis from three phase 1-2 trials, including 54 patients with advanced or metastatic disease presenting with an NTRK fusion, showed an objective response of $57 \%$ for the overall population, with $7 \%$ of patients achieving a complete response with a median PFS of 11.2 months and a median OS of 21 months ${ }^{8,41}$.

\section{NTRK fusions in breast cancer}

NTRK fusions represent a rare molecular alteration in breast cancer. Ross et al. identified only 16 tumors $(0.13 \%)$ with NTRK gene fusions among 12,214 locally aggressive, relapsed, or metastatic BC using comprehensive genomic profiling. Among them, nine cases were ductal carcinomas and three were secretory carcinomas. All tumors were HER2 negative, more commonly TNBC, and the majority had NTRK1 fusions ${ }^{42}$.

Conversely, human secretory breast carcinoma represents a rare subtype of invasive carcinoma (less than $0.02 \%$ of all breast cancers $)^{43}$ described in pediatric and adult populations that very frequently (above 90\%) harbor ETV6-NTRK3 gene fusion ${ }^{44}$ Genomic profiling of secretory breast carcinoma showed that most cases are classified as basal-like tumors with triple-negative receptor status ${ }^{45,46}$. However, ETV6-NTRK3 gene fusion is usually associated with indolent, slow-growing tumors with favorable prognosis, highlighting the molecular heterogeneity of triplenegative tumors ${ }^{47,48}$. A small series of 24 cases of secretory breast carcinoma evaluating pan-TRK IHC as a diagnostic assay found a positivity expression rate of $95.8 \%$. A pattern of diffuse and/or at least focally strong nuclear staining was a sensitive $(83.3 \%)$ and specific (100\%) diagnostic marker for this entity and may provide a more rapid and cost-effective test than FISH or NGS ${ }^{49}$.

To date, there is information about the efficacy of these agents in 15 patients with $\mathrm{MBC}$, with response rates of approximately $80 \%$ (Table 1$)^{8,40,50}$. Tumors known to have a high frequency of NTRK fusions, such as secretory breast carcinoma, should always be evaluated for the presence of this molecular driver using RTPCR or FISH as initial methods. For tumors with a low pretest probability of a positive result (e.g., breast cancers in general), a cancer gene panel would be a better test to exclude other more common targetable molecular alterations (Fig. 2). Treatment of MBC harboring NTRK fusions with TRK inhibitors is approved for patients with progressive disease despite previous treatment and conveys impressive response and survival rates in initial studies. Further research should address the role of TRKi in earlier lines of therapy, especially in NTRK-enriched histologies.

\section{TUMOR MUTATIONAL BURDEN}

TMB can be defined as the total number of somatic mutations per megabase (mut/Mb) of the genome examined ${ }^{51,52}$. Tumors with high TMB have a high neoantigen burden, some of which might increase T-cell reactivity ${ }^{53}$. Thus, it was hypothesized that tumors with high TMB are more responsive to immune checkpoint inhibitors (ICls). In fact, several retrospective and prospective studies have suggested that high TMB is associated with improved response to ICls in several tumor types ${ }^{54-57}$.

The predictive role of TMB in the benefit of $\mathrm{ICls}$ has been surrounded by controversy. This can be partially explained by the fact that TMB has been calculated using different platforms. In addition, TMB is influenced by tumor purity, ploidy, sequencing depth of coverage, and analytic methodologies. Furthermore, the threshold definition of high TMB is still not optimized across cancer types ${ }^{58}$. Additionally, while the use of large panels (covering $>1.1$ megabase of the sequenced coding region) has been validated in the context of clinical trials, it is important to highlight that their use tends to overestimate the mutational burden compared with whole exome sequencing. Multiple ongoing initiatives are attempting to standardize TMB assessment, and further work is necessary to establish the best 
cut-off for using TMB as a predictive biomarker of response to immunotherapy ${ }^{51,53,59}$.

\section{How to determine TMB in clinical practice}

Different large NGS panels are approved/authorized by the FDA and provide TMB evaluation: (1) tissue-based FoundationOne CDx (F1CDx), which defines TMB as the number of base substitutions (including synonymous mutations) in the coding regions of targeted genes; ${ }^{60}$ (2) tissue-based MSK-IMPACT, which tabulates nonsynonymous mutations using data from both tumor and germline DNA; ${ }^{.55}$ (3) liquid biopsy-based Guardant360; and (4) the liquid-based biopsy FoundationOne Liquid $C D x^{61}$.

\section{Approval of Pembrolizumab for treating TMB-H cancers}

In June 2020, the FDA expanded the approval of pembrolizumab to include unresectable or metastatic tumors with TMB-H $(\geq 10$ mut/Mb) that have progressed following prior treatment and that have no satisfactory alternative therapy options. The FDA also approved the FoundationOneCDx assay as a companion diagnostic for pembrolizumab ${ }^{5}$. The approval was based on a preplanned retrospective analysis of KEYNOTE-158. Among 790 patients who received pembrolizumab and had sufficient tissue for TMB analysis, 102 (13\%) were identified as having TMB-H tumors. The ORR was $29 \%$ for TMB $\geq 10$ mut/Mb compared to $6 \%$ for the non-TMB-H group and was maintained even when MSI-H tumors were excluded. The median duration of response was not reached ${ }^{5}$.

\section{TMB in breast cancer}

Using publicly available genomic data from 3969 patients with breast cancer, Barroso-Sousa et al. showed that while the median TMB of these tumors was 2.63 mut/Mb, $5 \%$ of breast cancers had high TMB (cut-off of $\geq 10$ mut/Mb), and metastatic tumors had a greater prevalence of high TMB than primary tumors $(8.4 \%$ vs. $2.9 \%)$. TNBC had a significantly higher median TMB than hormone receptor-positive or HER-2-positive cancer, although the frequency of tumors with high TMB did not differ between subtypes. In addition, it was shown that high TMB tumors had higher RNA expression of the CD8-positive T-cell effectors GZMA and PRF1 with greater immune cytolytic activity, suggesting that these patients are more likely to respond to ICI therapies ${ }^{62}$. Interestingly, enrichment of hypermutation was significantly higher in metastatic invasive lobular carcinoma than in invasive ductal metastatic tumors, a finding also described by Sokol and colleagues ${ }^{62,63}$. Importantly, other studies confirmed that the prevalence of TMB-H in breast cancer is approximately $10 \%$, which is far from being a rare alteration ${ }^{64}$. Although the TMB-H threshold is still debated, the cut-off of $\geq 10$ mut/Mb was the most used in studies evaluating TMB in breast cancer ${ }^{62,65-67}$.

Given that MMR defects represent an important mechanism leading to hypermutation, it would be of interest to address whether there is an overlap between high TMB and MSI status in breast cancer. However, there is a paucity of data on this issue. Similar to Chumsri67 et al., our group previously showed that the APOBEC signature is the most common dominant mutational process associated with hypermutation in breast cancer, followed by the dMMR signatures ${ }^{62}$. The APOBEC family has enzymatic activity that is essential for innate and adaptive immune responses, and its upregulation or mutation can be used as a potential predictive marker for immunotherapy responses in nonsmall cell lung cancer ${ }^{68}$.

With respect to the role of TMB as a biomarker, one study demonstrated that TMB-H was an independent prognostic factor, with longer OS observed in HER2-positive MBC previously treated with standard treatment ${ }^{69}$. In mTNBC patients treated with antiPD-1/L1 therapies, Barroso-Sousa and colleagues observed a significant positive association of TMB-H with longer progression-free survival (PFS) independent of clinical factors and PD-L1 status, and there was a trend toward improved $\mathrm{OS}^{70}$.

To date, the only study designed to prospectively evaluate the use of ICls in patients with MBC and high TMB was the phase II basket study TAPUR. In this study, a cohort of 28 patients with $\mathrm{MBC}$ and $\mathrm{TMB}$ of $\geq 9$ received pembrolizumab. Of note, $93 \%$ of patients were treated with $\geq 3$ prior systemic therapies, and $46 \%$ were TNBC (Table 1). TMB-H ranged from 9 to 37 mut/Mb, disease control was obtained in $37 \%$ of patients, the ORR was $21 \%$, and the median PFS and OS were 10.6 and 30.6 weeks, respectively ${ }^{65}$.

An exploratory analysis from KEYNOTE-119-a randomized, open-label, phase III study of pembrolizumab monotherapy versus single-agent chemotherapy in previously treated $\mathrm{mTNBC}$ patients - revealed a prevalence of TMB- $\mathrm{H}$, defined as $\geq 10$ mut/Mb, of $10 \%$ in the evaluable population. A positive association was established among TMB and PFS and OS for patients treated with pembrolizumab but not for those who underwent chemotherapy ${ }^{66}$.

Data on TMB were also analyzed in patients included in the randomized, placebo-controlled, phase 3 trial Impassion130, which evaluated the combination of atezolizumab plus nabpaclitaxel or placebo plus nab-paclitaxel in patients with untreated mTNBC ${ }^{71}$. There was no correlation between TMB and PD-L1 status, and the prevalence of patients with TMB-H was $8 \%$. In this study, higher TMB levels were associated with improved PFS and OS in the atezolizumab versus placebo arm in patients with PD$\mathrm{L} 1$ + tumors but not in those with PD-L1- tumors. There was no information about the benefit specifically in the TMB-H ( $\geq 10$ mut/ $\mathrm{Mb}$ ) population.

Regardless of ICls (combined with chemotherapy) being approved for patients with PD-L1-positive MTNBC, it would be of clinical interest to investigate 1) whether patients with other breast cancer subtypes and $\mathrm{TMB}-\mathrm{H}$, such as estrogen receptorpositive tumors, could also benefit from $\mathrm{ICls}$ and 2) whether patients with TMB-H PD-L1-positive MTNBC could be treated solely with immunotherapy and be spared from chemotherapy. NIMBUS (NCT03789110), an ongoing phase 2 trial, is recruiting patients with HER2-negative MBC whose tumors have a $T M B \geq 9$ mut/Mb for treatment with a combination of nivolumab and ipilimumab ${ }^{72}$.

\section{TRANSLATING THESE DATA INTO CLINICAL PRACTICE}

To aid oncologists in defining the utility of target treatments according to specific molecular alterations, ESMO developed the ESCAT scale, classifying molecular drivers into six tiers (I-V and X) based on the level of clinical evidence and providing guidance for treatment with targeted therapies ${ }^{9}$. In 2019, Condorelli et al. classified molecular drives in BC according to this scale: MSI and NTRK were assigned to tier IC. In this category, a target is suitable for routine use, and the strength of evidence is based on clinical trials conducted in multiple tumor types or basket trials that demonstrated clinical benefit for the target-drug pair with a similar magnitude of benefit across different tumor types ${ }^{73}$. With regard to $\mathrm{TMB}$, there is no formal ESCAT designation from the breast cancer panel.

While searching for these particular molecular alterations in $\mathrm{MBC}$, it is important to consider aspects including the prevalence of the molecular driver, accuracy, cost, and availability of different detection methods and reimbursement or regional regulatory policies regarding access to treatment. Although NGS panels are increasingly being used and able to identify multiple drivers at the same time, their costs are still not affordable for many patients or not covered by some payers. Additionally, patients must be aware that the chance of finding a druggable alteration is low. At the present time, some guidelines do not recommend NGS for advanced breast cancer in routine clinical practice ${ }^{74,75}$. On the 
other hand, it is important to highlight that FDA tissue-agnostic approvals have focused on patients who have progressed following prior lines of treatment and have no satisfactory alternative therapeutic options in the metastatic setting. Taking all these pros and cons into account, routinely performing tests for evaluating the status of these three biomarkers for patients with MBC is recommended if feasible (Fig. 2).

\section{FUTURE PERSPECTIVES}

It is becoming clear that for any target, there is a spectrum of actionability that extends from histology-specific to histologyagnostic biomarkers and that this degree of actionability has a dynamic nature that will need continuous revision with the emergence of new drugs and regimens ${ }^{76}$. This highlights the need for additional data on the efficacy of drugs targeting specific biomarkers in patients with advanced breast cancer.

Given their low frequency in breast cancer, a feasible alternative to obtaining more data on the effectiveness of agents targeting MSI-H/dMMR and NTRK fusions could be to obtain real-world data. An international registry capturing experience from physicians around the world would be of value in this situation. With regards to $\mathrm{TMB}-\mathrm{H}$, the scenario is different. The prevalence of TMB-H in MBC is approximately $10 \%$ (far from being considered a rare genomic event), and it would be desirable to generate prospective, randomized data on the effectiveness of immunotherapy in MBC patients, similar to what has been done in other rare molecular drivers such as ALK rearrangements ${ }^{77}$ or RET fusion $^{78}$ in nonsmall cell lung cancer.

In addition, other molecular alterations have potential for histology-agnostic designation, including RET alterations, BRAF mutations, fibroblast growth factor receptor (FGRF) aberrations, KRAS 12 G, ROS1, ALK, NRG1, HER2, and POLE/POLD1 ${ }^{76,79,80 .}$

\section{CONCLUSION}

The emergence of tissue-agnostic therapy in oncology is the apex of precision oncology. Since 2017, the FDA has approved the use of three different agents for tumor-agnostic treatment: pembrolizumab for patients with microsatellite instability or high tumor mutational burden, larotrectinib, and entrectinib-both for use in patients harboring tumors with NTRK fusions. However, only a few patients with $M B C$ were included in the pivotal trials that led to FDA approval of these agents. Despite the limitations regarding the low representation of patients with $M B C$ in pivotal studies, we recommend routinely performing tests to evaluate the status of these three biomarkers among patients with $M B C$, if feasible. However, given that the spectrum of actionability of these drivers can vary according to the tumor type, additional data assessing the degree of benefit and optimal treatment sequence in patients with $\mathrm{MBC}$ harboring these genomic alterations are needed.

Received: 3 March 2021; Accepted: 20 August 2021; Published online: 13 September 2021

\section{REFERENCES}

1. Sung, $H$. et al. Global cancer statistics 2020: GLOBOCAN estimates of incidence and mortality worldwide for 36 cancers in 185 countries. CA Cancer J. Clin 71, 209 (2021).

2. Cardoso, F. et al. Global analysis of advanced/metastatic breast cancer: decade report (2005-2015). Breast 39, 131-138 (2018).

3. Pestana, R. C., Sen, S., Hobbs, B. P. \& Hong, D. S. Histology-agnostic drug development - considering issues beyond the tissue. Nat. Rev. Clin. Oncol. 17 555-568 (2020)

4. Seligson, N. D., Knepper, T. C., Ragg, S. \& Walko, C. M. Developing drugs for tissueagnostic indications: a paradigm shift in leveraging cancer biology for precision medicine. Clin. Pharmacol. Ther 109, 334 (2021).
5. Keytruda [package insert]. (Merck, Whitehouse Station, NJ, 2019).

6. Marabelle, A. et al. Efficacy of pembrolizumab in patients with noncolorectal high microsatellite instability/mismatch repair-deficient cancer: results from the phase II KEYNOTE-158 study. J. Clin. Oncol. 38, 1-10 (2019).

7. Drilon, A. et al. Efficacy of larotrectinib in TRK fusion-positive cancers in adults and children. N. Engl. J. Med. 378, 731-739 (2018).

8. Doebele, R. C. et al. Entrectinib in patients with advanced or metastatic NTRK fusion-positive solid tumours: integrated analysis of three phase 1-2 trials. Lancet Oncol. 21, 271-282 (2020).

9. Mateo, J. et al. A framework to rank genomic alterations as targets for cancer precision medicine: the ESMO Scale for Clinical Actionability of molecular Targets (ESCAT). Ann. Oncol. J. Eur. Soc. Med. Oncol. 29, 1895-1902 (2018).

10. Li, K., Luo, H., Huang, L., Luo, H. \& Zhu, X. Microsatellite instability: a review of what the oncologist should know. Cancer Cell Int. 20, 16 (2020).

11. Eso, Y., Shimizu, T., Takeda, H., Takai, A. \& Marusawa, H. Microsatellite instability and immune checkpoint inhibitors: toward precision medicine against gastrointestinal and hepatobiliary cancers. J. Gastroenterol. 55, 15-26 (2020).

12. Marcus, L., Lemery, S. J., Keegan, P. \& Pazdur, R. FDA approval summary: pembrolizumab for the treatment of microsatellite instability-high solid tumors. Clin. Cancer Res. 25, 3753-3758 (2019).

13. Luchini, C. et al. ESMO recommendations on microsatellite instability testing for immunotherapy in cancer, and its relationship with PD-1/PD-L1 expression and tumour mutational burden: a systematic review-based approach. Ann. Oncol. 30, 1232-1243 (2019).

14. Suraweera, N. et al. Evaluation of tumor microsatellite instability using five quasimonomorphic mononucleotide repeats and pentaplex PCR. Gastroenterology 123, 1804-1811 (2002).

15. Yoshino, T. JSCO-ESMO-ASCO-JSMO-TOS: international expert consensus recommendations for tumour-agnostic treatments in patients with solid tumours with microsatellite instability or NTRK fusions. Ann. Oncol. 31, 12 (2020).

16. Goel, A., Nagasaka, T., Hamelin, R. \& Boland, C. R. An optimized pentaplex PCR for detecting DNA mismatch repair-deficient colorectal cancers. PLOS ONE 5, e9393 (2010).

17. Kok, M., Chalabi, M. \& Haanen, J. How I treat MSI cancers with advanced disease. ESMO Open 4, e000511 (2019).

18. Nowak, J. A. et al. Detection of mismatch repair deficiency and microsatellite instability in colorectal adenocarcinoma by targeted next-generation sequencing. J. Mol. Diagn. 19, 84-91 (2017).

19. Le, D. T. et al. PD-1 blockade in tumors with mismatch-repair deficiency. N. Engl. J. Med. 372, 2509-2520 (2015).

20. Le, D. T. et al. Mismatch repair deficiency predicts response of solid tumors to PD1 blockade. Science 357, 409 (2017).

21. Bonneville, R. et al. Landscape of microsatellite instability across 39 cancer types. JCO Precis. Oncol. 2017, 1-15 (2017).

22. Cortes-Ciriano, I., Lee, S., Park, W.-Y., Kim, T.-M. \& Park, P. J. A molecular portrait of microsatellite instability across multiple cancers. Nat. Commun. 8, 15180 (2017).

23. Adem, C. et al. Microsatellite instability in hereditary and sporadic breast cancers. Int. J. Cancer 107, 580-582 (2003).

24. Fusco, N. et al. Mismatch repair protein loss as a prognostic and predictive biomarker in breast cancers regardless of microsatellite instability. JNCI Cancer Spectr. 2 (2018).

25. Siah, S. P. et al. Microsatellite instability markers in breast cancer: a review and study showing MSI was not detected at 'BAT 25 ' and 'BAT 26 ' microsatellite markers in early-onset breast cancer. Breast Cancer Res. Treat. 60, 135-142 (2000).

26. Kurata, K. et al. Microsatellite instability in Japanese female patients with triplenegative breast cancer. Breast Cancer 27, 490-498 (2020).

27. Wen, Y. H. DNA mismatch repair deficiency in breast carcinoma: a pilot study of triple-negative and non-triple-negative tumors. Am. J. Surg. Pathol 36, 1700 (2012).

28. Horimoto, Y. et al. Microsatellite instability and mismatch repair protein expressions in lymphocyte-predominant breast cancer. Cancer Sci. 111, 2647-2654 (2020).

29. Lacroix-Triki, M. et al. Absence of microsatellite instability in mucinous carcinomas of the breast. Int. J. Clin. Exp. Pathol. 4, 22-31 (2011).

30. Vaishnavi, A., Le, A. T. \& Doebele, R. C. TRKing down an old oncogene in a new era of targeted therapy. Cancer Discov. 5, 25-34 (2015).

31. Cocco, E., Scaltriti, M. \& Drilon, A. NTRK fusion-positive cancers and TRK inhibitor therapy. Nat. Rev. Clin. Oncol. 15, 731-747 (2018).

32. Marchiò, $C$. et al. ESMO recommendations on the standard methods to detect NTRK fusions in daily practice and clinical research. Ann. Oncol. J. Eur. Soc. Med. Oncol. 30, 1417-1427 (2019).

33. Solomon, J. P., Benayed, R., Hechtman, J. F. \& Ladanyi, M. Identifying patients with NTRK fusion cancer. Ann. Oncol. J. Eur. Soc. Med. Oncol. 30, viii16-viii22 (2019).

34. Hechtman, J. F. et al. Pan-Trk immunohistochemistry is an efficient and reliable screen for the detection of NTRK fusions. Am. J. Surg. Pathol. 41, 1547-1551 (2017). 
35. Rudzinski, E. R. et al. Pan-Trk immunohistochemistry identifies NTRK rearrangements in pediatric mesenchymal tumors. Am. J. Surg. Pathol. 42, 927-935 (2018).

36. Gatalica, Z., Xiu, J., Swensen, J. \& Vranic, S. Molecular characterization of cancers with NTRK gene fusions. Mod. Pathol. 32, 147-153 (2019).

37. Chiang, S. et al. NTRK fusions define a novel uterine sarcoma subtype with features of fibrosarcoma. Am. J. Surg. Pathol. 42, 791-798 (2018).

38. Solomon, J. P. et al. NTRK fusion detection across multiple assays and 33,997 cases: diagnostic implications and pitfalls. Mod. Pathol. 33, 38-46 (2020).

39. Vitrakvi [package insert]. (Loxo Oncology, Inc., Stamford, CT, 2019).

40. Hong, D. S. et al. Larotrectinib in patients with TRK fusion-positive solid tumours: a pooled analysis of three phase $1 / 2$ clinical trials. Lancet Oncol. 21, 531-540 (2020).

41. Rozlytrek [package insert]. (Genentech, Inc., South San Francisco, CA, 2019).

42. Ross, J. S. et al. Abstract P2-09-15: NTRK fusions in breast cancer: Clinical, pathologic and genomic findings. Cancer Res. 78, P2-P2-09-P2-P2-015 (2018).

43. Jacob, J. D. et al. Rare breast cancer: 246 invasive secretory carcinomas from the National Cancer Data Base. J. Surg. Oncol. 113, 721-725 (2016).

44. Tognon, C. et al. Expression of the ETV6-NTRK3 gene fusion as a primary event in human secretory breast carcinoma. Cancer Cell 2, 367-376 (2002).

45. Krings, G. et al. Genomic profiling of breast secretory carcinomas reveals distinct genetics from other breast cancers and similarity to mammary analog secretory carcinomas. Mod. Pathol. 30, 1086-1099 (2017).

46. Laé, M. et al. Secretory breast carcinomas with ETV6-NTRK3 fusion gene belong to the basal-like carcinoma spectrum. Mod. Pathol. 22, 291-298 (2009).

47. Horowitz, D. P., Sharma, C. S., Connolly, E., Gidea-Addeo, D. \& Deutsch, I. Secretory carcinoma of the breast: results from the survival, epidemiology and end results database. Breast Edinb. Scotl. 21, 350-353 (2012).

48. Hoda, R. S. et al. Secretory carcinoma of the breast: clinicopathologic profile of 14 cases emphasising distant metastatic potential. Histopathology 75, 213-224 (2019).

49. Harrison, B. T. et al. Pan-TRK immunohistochemistry: a useful diagnostic adjunct for secretory carcinoma of the breast. Am. J. Surg. Pathol. 43, 1693-1700 (2019).

50. Meric-Bernstam, F. et al. Abstract P6-20-02: activity of larotrectinib, a highly selective inhibitor of tropomyosin receptor kinase, in TRK fusion breast cancers. Cancer Res. 79, P6-P6-20-P6-P6-202 (2019).

51. Chan, T. A. et al. Development of tumor mutation burden as an immunotherapy biomarker: utility for the oncology clinic. Ann. Oncol. 30, 44-56 (2019).

52. Zehir, A. et al. Mutational landscape of metastatic cancer revealed from prospective clinical sequencing of 10,000 patients. Nat. Med. 23, 703-713 (2017).

53. Stenzinger, A. et al. Tumor mutational burden standardization initiatives: recommendations for consistent tumor mutational burden assessment in clinical samples to guide immunotherapy treatment decisions. Genes. Chromosomes Cancer 58, 578-588 (2019).

54. Goodman, A. M. et al. Tumor mutational burden as an independent predictor of response to immunotherapy in diverse cancers. Mol. Cancer Ther. 16, 2598-2608 (2017).

55. Samstein, R. M. et al. Tumor mutational load predicts survival after immunotherapy across multiple cancer types. Nat. Genet. 51, 202-206 (2019).

56. Hellmann, M. D. et al. Nivolumab plus ipilimumab in lung cancer with a high tumor mutational burden. N. Engl. J. Med. 378, 2093-2104 (2018).

57. Johnson, D. B. et al. Targeted next generation sequencing identifies markers of response to PD-1 blockade. Cancer Immunol. Res. 4, 959-967 (2016).

58. Legrand, F. A. et al. Association of high tissue TMB and atezolizumab efficacy across multiple tumor types. J. Clin. Oncol. 36, 12000-12000 (2018).

59. Merino, D. M. et al. Establishing guidelines to harmonize tumor mutational burden (TMB): in silico assessment of variation in TMB quantification across diagnostic platforms: phase I of the Friends of Cancer Research TMB Harmonization Project. J. Immunother. Cancer 8, e000147 (2020).

60. Marabelle, A. et al. Association of tumour mutational burden with outcomes in patients with advanced solid tumours treated with pembrolizumab: prospective biomarker analysis of the multicohort, open-label, phase 2 KEYNOTE-158 study. Lancet Oncol. 21, 1353-1365 (2020).

61. Woodhouse, R. et al. Clinical and analytical validation of FoundationOne Liquid CDx, a novel 324-Gene cfDNA-based comprehensive genomic profiling assay for cancers of solid tumor origin. PloS ONE 15, e0237802 (2020).

62. Barroso-Sousa, R. et al. Prevalence and mutational determinants of high tumor mutation burden in breast cancer. Ann. Oncol. 31, 387-394 (2020).

63. Sokol, E. S. et al. Loss of function of NF1 is a mechanism of acquired resistance to endocrine therapy in lobular breast cancer. Ann. Oncol. 30, 115-123 (2019).

64. Bertucci, F. et al. Genomic characterization of metastatic breast cancers. Nature 569, 560-564 (2019).

65. Alva, A. S. et al. Pembrolizumab (P) in patients (pts) with metastatic breast cancer (MBC) with high tumor mutational burden (HTMB): Results from the Targeted Agent and Profiling Utilization Registry (TAPUR) Study. J. Clin. Oncol. 37, 1014-1014 (2019).
66. Winer, E. P. et al. Association of tumor mutational burden (TMB) and clinical outcomes with pembrolizumab (pembro) versus chemotherapy (chemo) in patients with metastatic triple-negative breast cancer (mTNBC) from KEYNOTE119. J. Clin. Oncol. 38, 1013-1013 (2020).

67. Chumsri, S. et al. Durable complete response with immune checkpoint inhibitor in breast cancer with high tumor mutational burden and APOBEC signature. J. Natl Compr. Cancer Netw. JNCCN 18, 517-521 (2020).

68. Wang, S., Jia, M., He, Z. \& Liu, X.-S. APOBEC3B and APOBEC mutational signature as potential predictive markers for immunotherapy response in non-small cell lung cancer. Oncogene 37, 3924-3936 (2018).

69. Park, S. E. et al. Clinical implication of tumor mutational burden in patients with HER2-positive refractory metastatic breast cancer. Oncolmmunology 7, e1466768 (2018).

70. Barroso-Sousa, R. et al. Tumor mutational burden and PTEN alterations as molecular correlates of response to PD-1/L1 blockade in metastatic triplenegative breast cancer. Clin. Cancer Res. 26, 2565-2572 (2020).

71. Emens, L. A. et al. 296P Tumour mutational burden and clinical outcomes with first-line atezolizumab and nab-paclitaxel in triple-negative breast cancer: Exploratory analysis of the phase III IMpassion 130 trial. Ann. Oncol. 31, S360-S361 (2020).

72. Barroso-Sousa, R. et al. Nimbus: A phase II study of nivolumab plus ipilimumab in metastatic hypermutated HER2-negative breast cancer. J. Clin. Oncol. 37, TPS1115-TPS1115 (2019).

73. Condorelli, R. et al. Genomic alterations in breast cancer: level of evidence for actionability according to ESMO Scale for Clinical Actionability of molecular Targets (ESCAT). Ann. Oncol. J. Eur. Soc. Med. Oncol. 30, 365-373 (2019).

74. Cardoso, F. et al. 5th ESO-ESMO international consensus guidelines for advanced breast cancer (ABC 5)†. Ann. Oncol. 31, 1623 (2020).

75. Mosele, F. et al. Recommendations for the use of next-generation sequencing (NGS) for patients with metastatic cancers: a report from the ESMO Precision Medicine Working Group. Ann. Oncol. J. Eur. Soc. Med. Oncol. 31, 1491-1505 (2020).

76. Tarantino, P., Mazzarella, L., Marra, A., Trapani, D. \& Curigliano, G. The evolving paradigm of biomarker actionability: histology-agnosticism as a spectrum, rather than a binary quality. Cancer Treat. Rev. https://doi.org/10.1016/j.ctrv.2021.102169 (2021).

77. Shaw, A. T. et al. First-line lorlatinib or crizotinib in advanced ALK-positive lung cancer. N. Engl. J. Med. 383, 2018-2029 (2020).

78. Solomon, B. J. et al. Phase III study of selpercatinib versus chemotherapy \pm pembrolizumab in untreated RET positive non-small-cell lung cancer. Future Oncol. Lond. Engl. 17, 763-773 (2021).

79. Subbiah, V. \& Cote, G. J. Advances in targeting RET-dependent cancers. Cancer Discov. 10, 498-505 (2020).

80. Holderfield, M., Deuker, M. M., McCormick, F. \& McMahon, M. Targeting RAF kinases for cancer therapy: BRAF-mutated melanoma and beyond. Nat. Rev. Cancer 14, 455-467 (2014).

\section{AUTHOR CONTRIBUTIONS}

L.N.W. contributed to the literature search, conception and design of the article and drafted the first version of the manuscript. S.M.T. and C.H.B. provided critical revisions of the manuscript. R.B.-S. contributed to the conception and design of the article and provided critical revisions of the manuscript. All authors approved the final submitted work.

\section{COMPETING INTERESTS}

S.M.T. receives institutional research funding from AstraZeneca, Lilly, Merck, Nektar, Novartis, Pfizer, Genentech/Roche, Immunomedics, Exelixis, Bristol-Myers Squibb, Eisai, Nanostring, Cyclacel, Odonate, and Seattle Genetics; has served as an advisor/ consultant to AstraZeneca, Lilly, Merck, Nektar, Novartis, Pfizer, Genentech/Roche, Immunomedics, Bristol-Myers Squibb, Eisai, Nanostring, Puma, Sanofi, Celldex, Paxman, Puma, Silverback Therapeutics, G1 Therapeutics, AbbVie, Anthenex, OncoPep, Outcomes4Me, Kyowa Kirin Pharmaceuticals, Daiichi-Sankyo, and Samsung Bioepsis Inc. C.H.B.: Grants/research support: (to the institution) Pfizer, Novartis, Amgen, AstraZeneca, Boehringer Ingelheim, GlaxoSmithKline, Roche/Genentech, Lilly, Sanofi, Taiho Pharmaceutical, Mylan, Merrimack, Merck, AbbVie, Astellas Pharma, BioMarin, Bristol-Myers Squibb, Daiichi Sankyo, Abraxis Biosciences, AB Science, Asana Biosciences, Medivation, Exelixis, ImClone Systems, LEO Pharma, Millennium, Merck KGaA, Shanghai Henlius Biotech, Polyphor, PharmaMar. Ownership or Stocks: Biomarker, Tummi, MEDSir. Advisory Boards and Consulting: Boehringer-Ingelheim, GSK, Novartis, Pfizer, Roche/Genentech, Eisai, Bayer, MSD, AstraZeneca, Zodiac, Lilly, Sanofi. R.B-S. has served as an advisor/consultant to Eli Lilly, Merck Sharp and Dohme, and Roche and has received honoraria from Bard Acess, Bristol-Myers Squib, Libbs, Eli 
Lilly, Novartis, Pfizer, Roche, Zodiac and travel accommodations or expenses from Roche and Daiichi-Sankyo. The remaining author declares no competing interests.

\section{ADDITIONAL INFORMATION}

Correspondence and requests for materials should be addressed to Romualdo Barroso-Sousa.

Reprints and permission information is available at http://www.nature.com/ reprints

Publisher's note Springer Nature remains neutral with regard to jurisdictional claims in published maps and institutional affiliations.
Open Access This article is licensed under a Creative Commons Attribution 4.0 International License, which permits use, sharing, adaptation, distribution and reproduction in any medium or format, as long as you give appropriate credit to the original author(s) and the source, provide a link to the Creative Commons license, and indicate if changes were made. The images or other third party material in this article are included in the article's Creative Commons license, unless indicated otherwise in a credit line to the material. If material is not included in the article's Creative Commons license and your intended use is not permitted by statutory regulation or exceeds the permitted use, you will need to obtain permission directly from the copyright holder. To view a copy of this license, visit http://creativecommons. org/licenses/by/4.0/.

(c) The Author(s) 2021 